\title{
Symptomatic Subcapsular and Perinephric Hematoma Following Ureteroscopic Lithotripsy for Renal Calculi
}

\author{
Michael A. Kozminski, MD, David J. Kozminski, BA, William W. Roberts, MD, \\ Gary J. Faerber, MD, John M. Hollingsworth, MD, MS, and J. Stuart Wolf, Jr., MD
}

\begin{abstract}
Objective: Ureteroscopic lithotripsy (URSL) is believed to be associated with less risk of symptomatic renal hematoma than extracorporeal shockwave lithotripsy (SWL) and percutaneous nephrolithotomy (PCNL). We sought to document the rate of and risk factors for this rare complication following URSL for renal calculi. Methods: With Institutional Review Board approval, we reviewed 1087 cases of URSL performed between July 2009 and October 2012 for four surgeons. We identified cases for renal calculi complicated by symptomatic "hematoma" by searching electronic medical records of patients undergoing URSL with a web-based search tool and cross-referencing with a departmental quality improvement database for postoperative complications. Chi-squared tests were used to assess risk factors.

Results: Among 877 renal units exposed to URSL for renal calculi, 4 were complicated by symptomatic subcapsular hematomas $(\mathrm{SH})$ and 3 by symptomatic perinephric hematomas $(\mathrm{PH})$, yielding a $0.5 \%$ and $0.3 \%$ rate for each complication, respectively. Pain was the primary presenting symptom. Almost all cases presented within 24 to 48 hours postop. Two PH patients required postoperative blood transfusion. Four patients (two SH, two $\mathrm{PH}$ ) were hospitalized for observation. Ureteral sheaths were used in two cases (one $\mathrm{PH}$ and one $\mathrm{SH}$ ). There was no association with age, diabetes, body mass index (BMI), or operative duration ( $p$-values all $>0.05)$. However, hematoma did correlate with female gender, preoperative hypertension, preoperative ureteral stenting, intraoperative ureteral sheath use, and postoperative ureteral stenting (all $p$-values $<0.0001$ ).

Conclusions: While symptomatic hematoma is a complication of URSL, the rate of such outcome $(0.8 \%)$ is far less than that reported by prior series with SWL and PCNL. This may partially be attributable to collection biases, where subclinical cases are not imaged, or anchoring biases, where clinicians attribute symptoms to another possible etiology. This outcome can be morbid, but can often be conservatively managed with observation.
\end{abstract}

\section{Introduction}

$\mathbf{U}$ RETEROSCOPIC LITHOTRIPSY (URSL) with holmium:yttrium-aluminum-garnet (Ho:YAG) laser is considered an effective and safe method of managing renal stones. ${ }^{1}$ Endoscopic advances, including smaller caliber ureteroscopes with greater flexibility, have yielded relatively low complication rates for URSL. ${ }^{2}$ Major complications tend to be rare, ${ }^{3}$ making URSL preferable compared to percutaneous nephrolithotomy (PCNL) or extracorporeal shockwave lithotripsy (SWL) for more complex patients (e.g., obese, bleeding diatheses).

Through our practice, we have encountered anecdotal cases of symptomatic, postoperative renal bleeding following URSL, specifically perinephric hematoma (PH) and subcapsular hematoma (SH). This acute hemorrhage can have compressive effects on the renal parenchyma, at least theoretically compromising renal function and hemodynamics. ${ }^{4}$ It has been previously demonstrated that these account for only a small percentage of postoperative sequelae and tend to occur far less frequently than in patients undergoing either PCNL or SWL. ${ }^{2,3}$ Consequently, the current literature focuses heavily on outcomes from these latter two stone treatments. ${ }^{5-9}$ Only a handful of authors have reported on hematoma as a postoperative outcome in URSL, and fewer still have attempted to qualify risk factors for the development of $\mathrm{SH}$ and $\mathrm{PH} .^{2,10,11}$

For these reasons, we sought to determine our own institutional rate of this rare complication and to determine potential risk factors. We hypothesized, given previous studies, that symptomatic hematoma rates would be low, and management of hematoma patients could be conservative with observation.

Department of Urology, University of Michigan Medical School, Ann Arbor, Michigan. 


\section{Patients and Methods}

With approval from the University of Michigan Medical Institutional Review Board, we retrospectively reviewed our departmental database of ureteroscopic procedures performed between July 2009 and October 2012 by four surgeons (Fig. 1). In total, 1087 cases were identified for "lithotripsy." A web-based search tool ${ }^{12}$ was employed to identify which of these cases were complicated by symptomatic "hematoma" based on review of clinical care notes, operative dictations, and radiology reports. All of the identified symptomatic "hematoma" cases had documented PH or $\mathrm{SH}$ based on computed tomography or sonography as confirmed by finalized radiology dictations. This search was also cross-referenced with a departmental quality improvement database for postoperative complications to improve the sensitivity of our search for symptomatic cases. The remaining 1077 uncomplicated cases were individually reviewed, after which 15 cases were ultimately excluded from analysis for either use of holmium laser for an urothelial tumor, blood clot, or encrusted indwelling stent; endoscopy within a neobladder chimney; concomitant PCNL; or failure to use a laser. Of the remaining 1062, 120 patients had bilateral URSL. On initial analysis of the data, no patients who underwent URSL exclusively for ureteral calculi suffered $\mathrm{SH}$ or PH. Since our presumption was that work in the kidney, not the ureter, results in the hematoma, we finally excluded all cases involving only ureteral calculi, which yielded a total of 877 renal units that were exposed to URSL for renal calculi.

Electronic medical records were reviewed for perioperative data. Patient-specific demographics, including age, sex, body mass index, preoperative hypertension, and diabetes mellitus were taken from preop anesthesia evaluations. Operative dictations were individually reviewed for stone laterality, stone location, and ureteral sheath/stent utilization. Operative duration was calculated from anesthesia records and intraoperative nursing reports.

All patients were counseled and informed consent was obtained preoperatively. Procedures were performed under general anesthesia in dorsal lithotomy position. Both Olympus semi-rigid, for distal ureteral calculi, and URF-P5 flexible, for proximal ureteral and renal calculi, ureteroscopes were utilized. A 200 or $300 \mu \mathrm{m}$ (for distal ureteral calculi) Ho:YAG laser fiber was used for lithotripsy, with the laser energy of 0.8 to $1.0 \mathrm{~J}$ and a frequency of 8 to $10 \mathrm{~Hz}$ (with modifications to these settings at the discretion of the surgeon). Normal saline irrigant was utilized in all cases with irrigation pressure at the discretion of the surgeon (usually 150-200 $\mathrm{mm} \mathrm{Hg}$, occasionally up to $300 \mathrm{~mm} \mathrm{Hg}$ ). The goal of lithotripsy either was to fragment all visualized stones to less than $1 \mathrm{~mm}$ in greatest diameter or to extract any fragments greater than $1 \mathrm{~mm}$. In general, with the former strategy, ureteral access sheaths were not employed; whereas with the latter strategy, they were used routinely. Percuflex double pigtail indwelling ureteral stents (Boston Scientific Corporation, Natick, MA), 6F, were postoperatively utilized based on surgeon's discretion. Generally, patients were discharged the same day as the procedure. Renal hematomaeither subcapsular or perinephric-was diagnosed after the presentation of symptoms or with adequate clinical suspicion, on the basis of either sonographic or computed tomographic imaging.

Potential risk factors were compared using bivariate analysis between patients with and without $\mathrm{PH}$ and $\mathrm{SH}$. Chi-squared tests were used to assess categorical data (gender, laterality, comorbidity status, sheath use, and stent use). For continuous variables (age, body mass index, and operation duration), data were ranked and then divided into two groups. The ratio of hematoma to no hematoma was then assessed using Chisquared tests. Both mean and median were utilized to divide the samples, but no difference was demonstrated between these stratifications. Stone location was analyzed using Pearson's chi-squared test. All tests were two-sided, and a $p$-value of less than 0.05 was considered statistically significant.

\section{Results}

We identified cases of symptomatic, postoperative renal bleeding following URSL, specifically PH and SH. Figure 2 demonstrates computed tomographic images of both the former (Fig. 2A), in which blood extravasates outside the renal capsule and into the retroperitoneal space, and the latter (Fig. 2B), were extravasated blood is retained within the renal capsule. Among 877 renal units analyzed, 4 were complicated by $\mathrm{SH}$ and 3 by $\mathrm{PH}$, yielding a $0.5 \%$ and $0.3 \%$ rate for each complication, respectively. None of these index patients were anticoagulated at the time of their procedures, aside from aspirin or nonsteroidal anti-inflammatory use. There was no
FIG. 1. Description of Cohort.

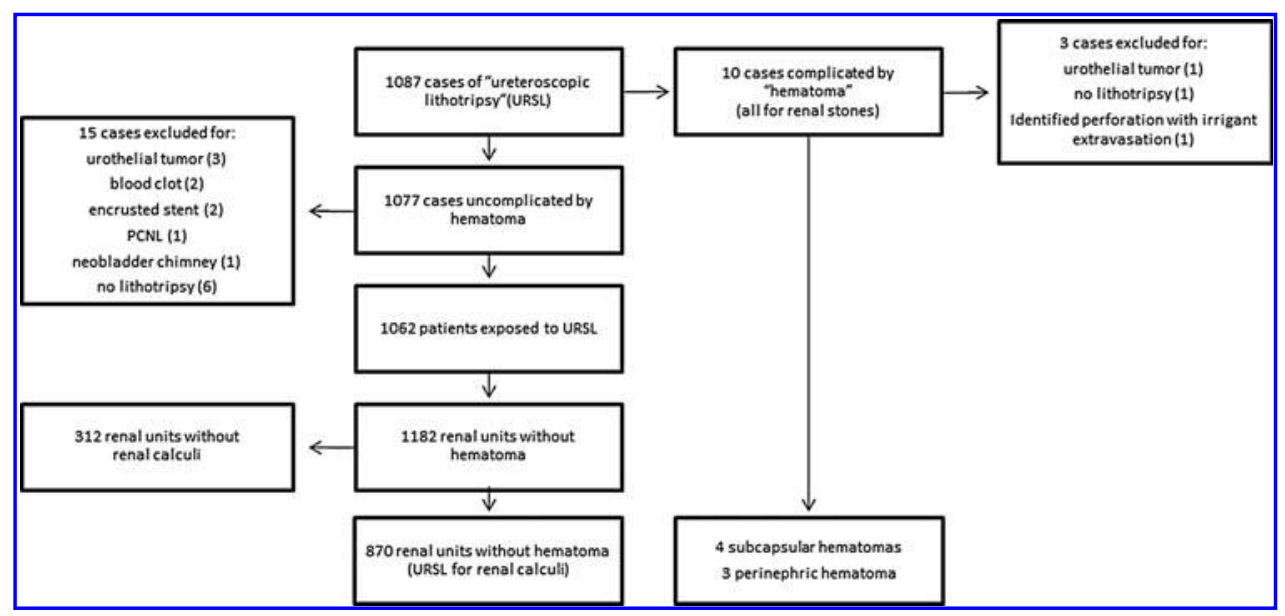



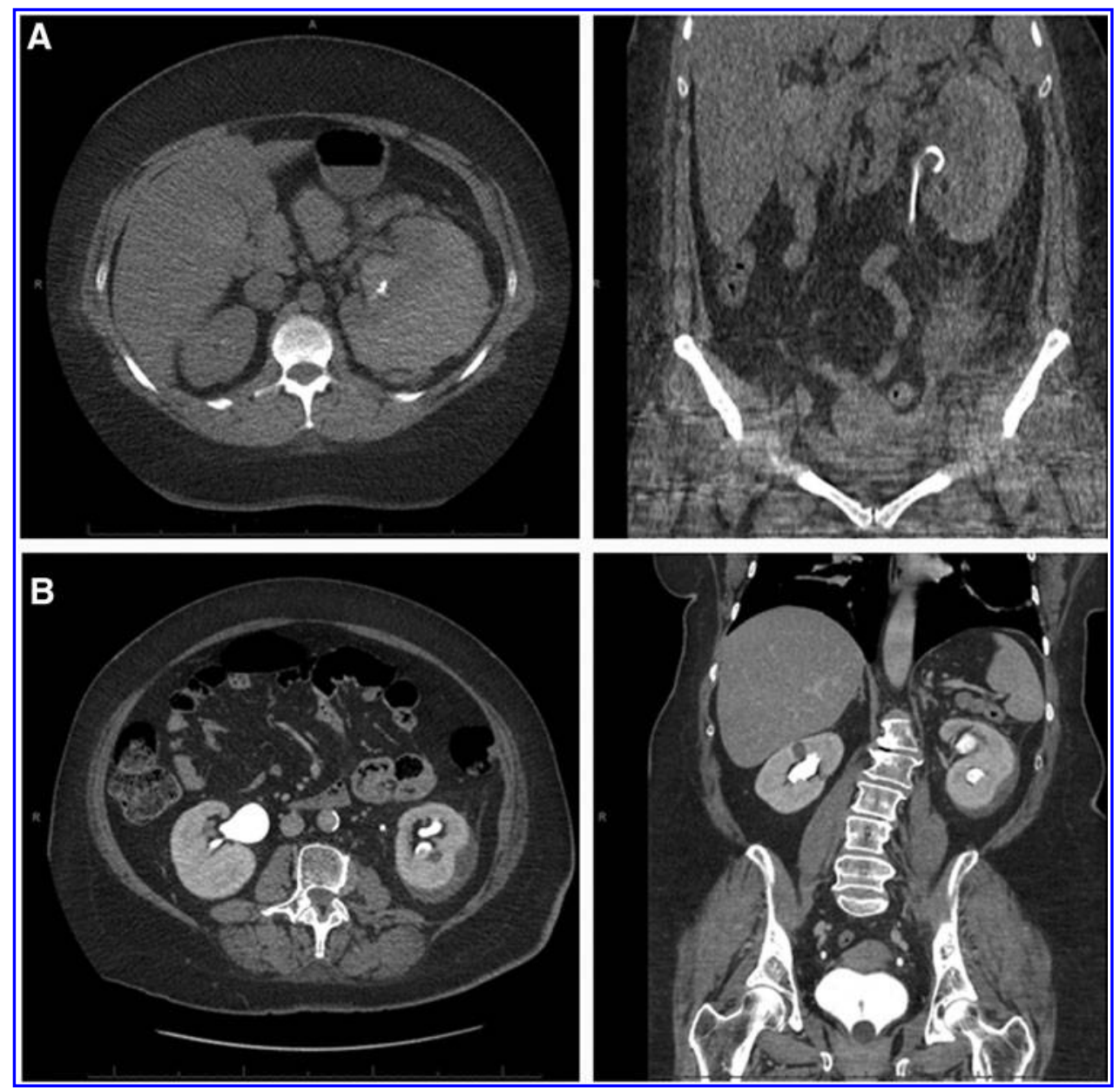

FIG. 2. Perinephric (A) and subcapsular hematomas (B).

association with age, diabetes mellitus, body mass index (BMI), or operative duration ( $p$-values all $>0.05$ ). However, hematoma did significantly correlate with female gender, preoperative hypertension (defined by preop anesthesia evaluation), preoperative ureteral stenting, intraoperative ureteral sheath use, and postoperative ureteral stenting (all $p$-values $<0.0001$ ) (Table 1).
Pain was the primary presenting symptom. Almost all cases presented within 24 to 48 hours postop. We did identify a single patient who presented $\sim 1$ month after surgery with nausea, weight loss, and midline abdominal pain. Two PH patients required postoperative blood transfusion. Four patients (two SH cases, and the two PH cases that required blood

Table 1. Bivariate Analyses of Perioperative Factors

\begin{tabular}{|c|c|c|c|c|}
\hline Characteristic & Total & Nonhematoma & Hematoma & p-Value \\
\hline Age (mean, SD) in years & $48.0(14.8)$ & $48.0(14.9)$ & $50.9(9.7)$ & 0.5 \\
\hline Gender ( $\%$ male $)$ & 47 & 47.2 & 14.3 & $<0.001$ \\
\hline Body mass index (mean, SD) & $31.2(10.1)$ & $31.1(10.1)$ & $39(17.0)$ & 0.6 \\
\hline \multicolumn{5}{|l|}{ Preop comorbidities $(\%)$} \\
\hline Diabetes mellitus & 19 & 19.1 & 14.3 & 0.2 \\
\hline Hypertension & 42.4 & 42.2 & 71.4 & $<0.001$ \\
\hline Stone laterality (\% left) & 50.6 & 50.8 & 57.1 & 0.2 \\
\hline Stone location $(n)$ & & & & 0.9 \\
\hline Kidney only & 741 & 735 & 6 & \\
\hline Ureter and kidney & 136 & 135 & 1 & \\
\hline Operation duration (min) & $43.4(26.5)$ & $43.3(26.6)$ & $47.7(16.8)$ & 0.4 \\
\hline Preop ureteral stent (\%) & 27.2 & 27.1 & 42.9 & $<0.001$ \\
\hline Intraop ureteral sheath (\%) & 12.8 & 12.6 & 28.6 & $<0.001$ \\
\hline Postop ureteral stent (\%) & 50.4 & 50.1 & 85.7 & $<0.001$ \\
\hline
\end{tabular}




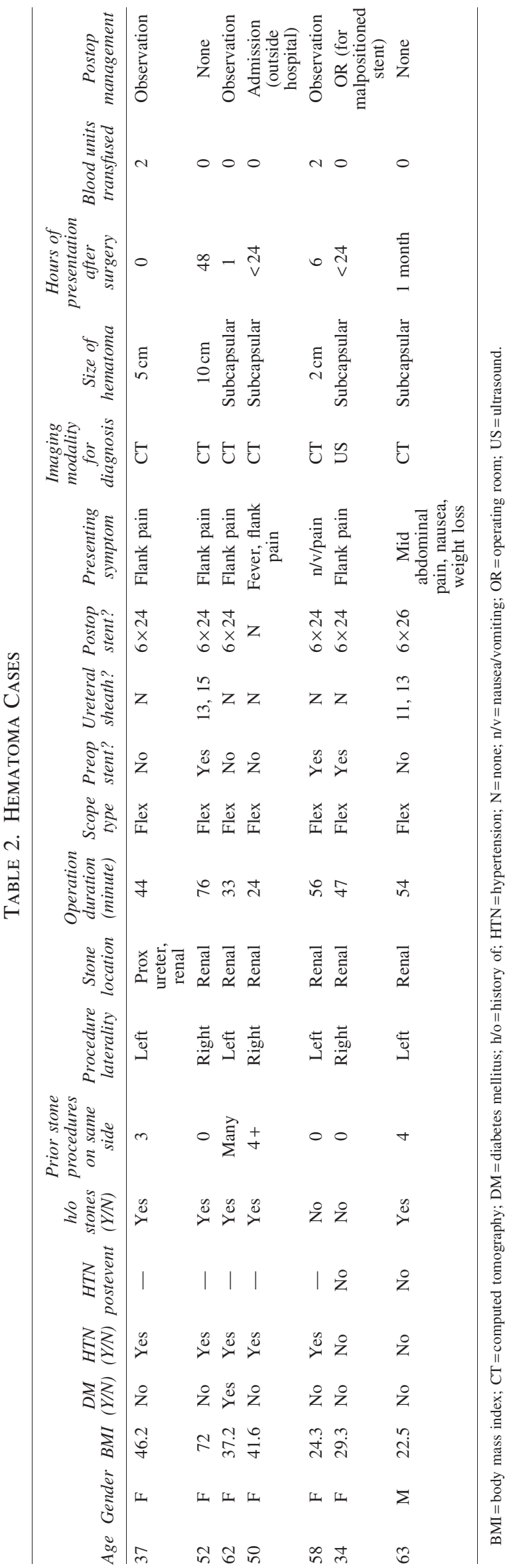

transfusions) were hospitalized. Ureteral access sheaths were used in two cases (one PH, one SH) (Table 2). None of our index cases seemed to develop ileus after developing a hematoma. Five of the seven patients had at least two prior URSL procedures on the ipsilateral kidney, and two of those five patients also had at least one PCNL on the affected side prior to their hematoma episode. Stone size in the index cases ranged from 1 up to $10 \mathrm{~mm}$ in greatest diameter. Five patients had multiple stones; the patients with solitary calculi had a stone diameter of $5 \mathrm{~mm}$. Stones were located in various locations, including the renal pelvis and both upper and lower poles. In terms of complicating intraoperative issues, two operative reports describe large clots within the renal collecting system (upper pole calyx in one case and renal pelvis in the other); another case had severe collecting system dilation with difficult visualization within the cavernous space; the stone was pushed into renal parenchyma in another case; one procedure included an infundibulotomy to access calculi; and another procedure included papillotomies in addition to lithotripsy.

\section{Discussion}

Through a retrospective review of 877 renal units, we defined the low incidence of symptomatic $\mathrm{PH}$ and $\mathrm{SH}$ after URSL for renal calculi. With bivariate analysis, we identified perioperative risk factors for this outcome. Specifically, we found that female gender, preoperative hypertension, preoperative ureteral stenting, intraoperative ureteral access sheath use, and postoperative ureteral stenting all have a significant association $(p<0.0001)$ with renal hematoma.

Understanding how these factors may increase the likelihood of developing postoperative hematoma is important for any urologist managing patients with renal calculi. Preoperative hypertension as a risk factor is consistent with prior experience, as this has also been found to increase the risk of retroperitoneal bleeding following SWL. ${ }^{5,6}$ Hypertension may increase the propensity to hemorrhage after URSLinduced injury or, in the case of hypertensive nephropathy, the renal tissue itself may have a greater tendency to damage. Female gender may relate to hormonal changes that can have an effect on ureteral peristalsis or pressure, but may also be an artifact secondary to our small number of events, especially given that most of the women who were found to have hematomas were peri- and postmenopausal. Interestingly, another recent retrospective analysis found a female predominance among their identified SH (six out of eight cases). ${ }^{13}$

The effect of ureteral stents and ureteral access sheaths on hematoma formation seems less intuitive, but these measures may instead be surrogates for other factors that are difficult to assess. For instance, preoperative stents are typically placed in the setting of obstruction or ureteral narrowing. These cases are frequently associated with some degree of hydronephrosis, and this distension may weaken the renal unit, making it more susceptible to pronounced bleeding. Unfortunately, many of our procedures are only preceded by KUB imaging (an abdominal x-ray focusing on the "kidneyureter-bladder"), which limits the assessment of pelvicaliectasis, especially in those patients who did not have a preoperative ureteral stent. Given institutional practice patterns, the use of intraoperative ureteral access sheaths is 
typically reserved for cases with a greater degree of stone burden and/or harder stones. This would seem to correlate with a greater degree of laser utilization and overall stone manipulation, which is difficult to objectively measure. Alternatively, the placement of the sheath itself may injure the renal parenchyma; however, this was never directly observed in any of our index cases. Finally, again based on institutional practice, postoperative stents are typically placed during cases that were prolonged, had intraoperative bleeding, had increased stone burden, or had ureteral narrowing or trauma that may have inhibited renal drainage following the operation. This could be another marker of case complexity, similar to intraoperative ureteral access sheath use, and the more significant factor could potentially be born out in a multivariable analysis, which we were unable to perform here given our low number of events. Our study is the first to implicate any of these variables as risk factors for post-USRL hematoma, and given the frequent use of pre and postoperative stenting and ureteral access sheath use in general, these data have immediate clinical relevance.

In previous case reports, the chief complaint of renal hematoma was flank pain. ${ }^{2,10,11,14}$ Our study corroborated this finding; those patients who were symptomatic all presented with pain. The delayed hematoma case was identified on computed tomography during evaluation of nausea, weight loss, and abdominal cramping. This patient required no postoperative intervention. Patients may also present with fever, palpable renal mass, increased creatinine, or decreased hemoglobin. Associated symptoms may include gross hematuria, diffused abdominopelvic pain, and hypotension. While prior reports have described interventions to evacuate the hematoma, ${ }^{2,11,14}$ we found that PH and SH can be conservatively managed with observation. Of our seven hematoma cases, only two required blood transfusions, one was admitted at an outside hospital, one was taken to the operating room for an incorrectly positioned ureteral stent, and none required surgery to drain the hematoma.

SH and PH are rare URSL postoperative outcomes. We found that together they occur in only $0.8 \%$ of URSL cases for renal calculi. Yet, this low rate may be partially attributable to certain biases. First, patients who experience minimally symptomatic or asymptomatic hematomas may not present for evaluation or undergo imaging to identify bleeding. We typically assess patients following URSL with KUB. As such, subclinical hematomas, if present, are likely to be undetected. Additionally, clinicians may succumb to anchoring bias, where the patient's symptoms are attributed to other etiologies (e.g., "stent pain") as opposed to the underlying hematoma. These phenomena may also result in an under diagnosis of post-USRL hematoma. However, as this study suggests, even symptomatic hematomas rarely require intervention, so there is unlikely to be a benefit to screening asymptomatic patients.

Our study may have other limitations. First, there were a number of factors that have been previously suggested as risk factors for $\mathrm{SH}$, including stone size and irrigation pressure, ${ }^{14}$ that were not assessed in our study since these are not consistently measured at our institution. However, we do not feel that stone size is a modifiable perioperative variable. One may argue that a patient with a larger stone size may be counseled toward PCNL rather than URSL. However, it is known that PCNL carries a higher risk of retroperitoneal hemorrhage, and for other surgical and/or medical reasons may not be preferred. Moreover, our institution uses inflatable pressurized infusion bags that only roughly approximate irrigant pressures. Higher irrigant pressures dilate the upper tract to a greater degree and potentially placed an increased stress on the given renal unit. However, in many cases, especially those complicated by poor visualization, higher irrigant pressures may be necessary to prevent mucosal injury during lithotripsy. Additionally, we did not include any laser parameters in our analysis, but again, these are typically adapted to treat the stone at hand, and the surgeon would be unlikely to change them intraoperatively to decrease hematoma risk in the face of inadequately treating the stone (and potentially increasing operative time/complexity).

\section{Conclusions}

While symptomatic hematoma is a complication of URSL for renal calculi, the rate of such outcome $(0.8 \%)$ is far less than that reported in other institutional series of SWL and PCNL. This may partially be attributable to various biases, all of which may result in under-diagnosis of post-URSL hematoma. This outcome can be morbid, but we believe can be conservatively managed with observation. Urologists can use this information in counseling patients preoperatively and guiding evaluation and management of patients who present with flank pain postoperatively.

\section{Disclosure Statement}

No competing financial interests exist.

\section{References}

1. Taie K, Jasemi M, Khazaeli D, et al. Prevalence and management of complications of ureteroscopy: A sevenyear experience with introduction of a new maneuver to prevent ureteral avulsion. Urol J 2012;9:356-360.

2. Chiu PK, Chan CK, Ma WK, et al. Subcapsular hematoma after ureteroscopy and laser lithotripsy. J Endourol 2013; 27:1115-1119.

3. Nuttall M, Abbaraju J, IK D, et al. A review of studies reporting on complications of upper urinary tract stone ablation using the holmium:YAG laser. Br J Med Surg Urol 2010;3:151-159.

4. Dopson SJ, Jayakumar S, Velez JC. Page kidney as a rare cause of hypertension: Case report and review of the literature. Am J Kidney Dis 2009;54:334-339.

5. Razvi H, Fuller A, Nott L, et al. Risk factors for perinephric hematoma formation after shockwave lithotripsy: A matched case-control analysis. J Endourol 2012;26: 1478-1482.

6. Lee HY, Yang YH, Shen JT, et al. Risk factors survey for extracorporeal shockwave lithotripsy-induced renal hematoma. J Endourol 2013;27:763-767.

7. Semins MJ, Bartik L, Chew BH, et al. Multicenter analysis of postoperative CT findings after percutaneous nephrolithotomy: Defining complication rates. Urology 2011; 78:291-294.

8. Rastinehad AR, Andonian S, Smith AD, et al. Management of hemorrhagic complications associated with percutaneous nephrolithotomy. J Endourol 2009;23:1763-1767. 
9. Chichakli R, Krause R, Voelzke B, et al. Incidence of perinephric hematoma after percutaneous nephrolithotomy. J Endourol 2008;22:1227-1232.

10. Duffey B, Lee J, Monga M. Perinephric hematoma following ureteroscopy and holmium laser lithotripsy. Open Urol Nephrol J 2008:36-37.

11. Bansal U, Sawant A, Dhabalia J. Subcapsular renal hematoma after ureterorenoscopy: An unknown complication of a known procedure. Urol Ann 2010;2:119-121.

12. Hanauer DA. EMERSE: The electronic medical record search engine. AMIA Annu Symp Proc 2006:941.

13. Meng H, Chen S, Chen G, et al. Renal subcapsular hemorrhage complicating ureterolithotripsy: An unknown complication of a known day-to-day procedure. Urol Int 2013;91:335-339.

14. Bai J, Li C, Wang S, et al. Subcapsular renal haematoma after holmium:yttrium-aluminum-garnet laser ureterolithotripsy. BJU Int 2012;109:1230-1234.
Address correspondence to: J. Stuart Wolf, Jr., MD

University of Michigan Medical School 1500 E. Medical Center Drive, Room 3875

Ann Arbor, MI 48109-5330

E-mail:wolfs@med.umich.edu

$\begin{aligned} & \text { Abbreviations Used } \\ & \mathrm{BMI}=\text { body mass index } \\ & \mathrm{Ho}: \mathrm{YAG}=\text { holmium:yttrium-aluminum-garnet } \\ & \mathrm{KUB}=\text { an abdominal x-ray focusing on the } \\ & \text { "kidney-ureter-bladder" } \\ & \mathrm{PCNL}=\text { percutaneous nephrolithotomy } \\ & \mathrm{PH}=\text { perinephric hematoma } \\ & \mathrm{SH}=\text { subcapsular hematomas } \\ & \mathrm{SWL}=\text { extracorporeal shockwave lithotripsy } \\ & \mathrm{URSL}=\text { ureteroscopic lithotripsy }\end{aligned}$

DOI: 10.1089/end.2014.0853

\title{
Editorial Comment for Kozminski et al.
}

\author{
Hassan Razvi, MD
}

$\mathbf{T}$ He AUTHORS PERFORMed a single institution retrospective review of 887 renal units subjected to ureteroscopic lithotripsy procedures for renal calculi to identify the rate and risk factors for the occurrence of symptomatic renal or perirenal hematomas. Seven patients were identified, including four in whom renal hematoms had developed and three with perinephric bleeds. All patients presented with pain, and all but one received a diagnosis within 48 hours of the procedure. Two patients needed a blood transfusion.

Potential risk factors for hematoma development were compared using bivariate analysis. Factors identified in the authors' analysis that correlated statistically with hematomas included female sex, preoperative hypertension, preoperative ureteral stent placement, the use of a ureteral access sheath, and postprocedure ureteral stent placement. Three of the seven patients involved intraoperative scenarios that may have precipitated renal bleeding, including a stone being pushed into the renal parenchyma, an infundibulotomy, and papillotomies.

The study methodology precludes the ability to prove causality for any of the possible risk factors assessed in this report. As the authors correctly state, the characteristics assessed may only be surrogates for as yet unrecognized additional factors.
What role do anatomic issues such as thin renal parenchyma or renal collecting system anomalies play? Could there be technical issues related to elevated intrarenal pressures generated by the irrigation fluid with subsequent barotrauma, or holmium laser shockwave-induced tissue effects? Does sudden decompression of an obstructed system lead to vascular changes that predispose to hemorrhage?

The authors are to be credited for raising awareness of a rarely described but possibly underreported phenomenon. Clearly, further attention should be directed to understanding the mechanisms and risk factors. This seems to be especially relevant as the application of flexible ureteroscopy continues to expand as a primary management option for intrarenal calculi.

\section{Address correspondence to: \\ Hassan Razvi, MD \\ Department of Urology \\ University of Western Ontario \\ London, Ontario \\ Canada}

E-mail: hrazvi@uwo.ca

Department of Urology, University of Western Ontario, London, Ontario, Canada. 


\section{This article has been cited by:}

1. Whitehurst Lily A. , MBBCh 1 and Somani Bhaskar K. , MRCS, FEBU, FRCS (Urol) 2 1Department of Urology, Royal Hampshire County Hospital, Winchester, United Kingdom. 2Department of Urology, University Hospital Southampton NHS Foundation Trust, Southampton, United Kingdom. . 2017. Perirenal Hematoma After Ureteroscopy: A Systematic Review. Journal of Endourology 31:5, 438-445. [Abstract] [Full Text HTML] [Full Text PDF] [Full Text PDF with Links]

2. Ming-Hong Kao, Chung-Cheng Wang. 2016. Risk factors for developing a perirenal hematoma after flexible ureteroscopic lithotripsy. Urological Science 27:3, 166-170. [CrossRef]

3. Petros Firas G., Zynger Debra L., Box Geoffrey N., Shah Ketul K.. 2016. Perinephric Hematoma and Hemorrhagic Shock as a Rare Presentation for an Acutely Obstructive Ureteral Stone with Forniceal Rupture: A Case Report. Journal of Endourology Case Reports 2:1, 74-77. [Abstract] [Full Text HTML] [Full Text PDF] [Full Text PDF with Links] 